\title{
Comparación de la profundidad de fotopolimerización de resinas fluidas a través de 4 porcelanas de alta opacidad
}

\section{Comparison of the light cure depth of flowable resins through 4 high-opacity porcelains}

\author{
Aranda $\mathrm{N}^{1}$, Aizencop $\mathrm{D}^{2}$, Ehrmantraut $\mathrm{M}^{2}$
}

\begin{abstract}
RESUMEN
Se estudia la profundidad de polimerización que se obtiene en resinas compuestas fluidas al ser iluminadas a través de bloques de cerámica de distinta composición y distintos grosores. En formadores metálicos cilíndricos de $4 \mathrm{~mm}$ de alto y $6 \mathrm{~mm}$ de diámetro se depositó resina fluida hasta enrasar en superficie y posteriormente se iluminó por $60 \mathrm{seg}$ interponiendo las distintas cerámicas entre la luz y la resina. Se utilizó una lámpara led para la iluminación del material. Algunos de los grupos en estudio presentaron diferencias significativas entre sí dependiendo del grosor de la cerámica y el tipo de ella. Es posible polimerizar resina fluida a través de cerámicas de distinta opacidad obteniendo grosores de resina mayores a los que se establecen para la línea de cementación de las restauraciones de cerámica libre de metal. Relevancia: A través de bloques de cerámica de diferente opacidad y composición se obtiene polimerización de resina compuesta fluida, lo que permitiría usar esta resina como medio de cementación de restauraciones a base de cerámicas cuyos grosores permitan el paso de la luz.
\end{abstract}

Rev. Clin. Periodoncia Implantol. Rehabil. Oral Vol. 6(3); 123-126, 2013.

Palabras clave: Porcelana dental, resinas fluidas, profundidad de polimerización.

\section{ABSTRACT}

We studied the depth of cure obtained in flowable composite resins when illuminated through ceramic blocks of different composition and different thicknesses. The flowable composite resin was deposited in forming metal cylinders of $4 \mathrm{~mm}$ of high and $6 \mathrm{~mm}$ of diameter, brought to volume and then illuminated for $60 \mathrm{sec}$ interposing the different ceramics between light and resin. A LED lamp was used to illuminate the material. Some of the groups exhibited significant differences among them depending on the thickness of the ceramic and its type. Flowable resin may polymerize through ceramics of different thicknesses and could be used for the cementation of ceramic restorations.

Rev. Clin. Periodoncia Implantol. Rehabil. Oral Vol. 6(3); 123-126, 2013.

Key words: Dental porcelain, flowable resin, depth of cure.

\section{INTRODUCCIÓN}

Las cerámicas actuales para prótesis fija, libre de metal, utilizan como base un tipo de ellas de gran opacidad $^{(1,2)}$, dificultando el paso de la luz que polimeriza el cemento a base de resina compuesta que fijará estas estructuras al diente.

Los cementos a base de resina compuesta que se utilizan para la fijación de las restauraciones de porcelana pueden ser activados, para su polimerización, de manera física, química o por ambos sistemas ${ }^{(3)}$.

Los de activación física por luz, son los que presentan las mejores propiedades, ya que este tipo de reacción es más eficiente en transformar monómero en polímero, resultando un material de mejor comportamiento mecánico, mayor dureza y mayor módulo elástico; no contiene láminas aromáticas en su composición, lo que permite gran estabilidad de color a través del tiempo ${ }^{(3,4)}$. Otra ventaja es el tiempo de trabajo útil, ya que no polimerizará hasta que se exponga a la luz que desencadenará su endurecimiento. Esta forma de activación deja muy poco monómero libre, disminuyendo la posibilidad de provocar daño pulpar o gingival. Como desventaja, este material foto activado no iniciará su polimerización si la cantidad de luz es insuficiente, situación que puede ocurrir al fijar restauraciones de mucho grosor, tal como ocurre cuando se interpone un material cerámico, como un vidrio ionómero, entre la fuente de luz y el material a polimerizar ${ }^{(5)}$.
Las otras formas de activación, química y dual no obtienen materiales con propiedades físico-mecánicas superiores, pero en zonas en que la luz no sea capaz de activar la resina, ésta puede realizarlo, ya que no necesitan energía lumínica para activarse ${ }^{(6)}$.

Estos materiales de fijación están compuestos a base de resina compuesta, que se diferencian de aquellas utilizadas para restauración directa, en que poseen menor cantidad de relleno, que resulta en un material de menores propiedades mecánicas. Como material alternativo existen las resinas compuestas fluidas (flow) que contienen mayor porcentaje de relleno inorgánico que los cementos a base de resina y ofrecen ventajas clínicas como mayor tiempo de trabajo, mejor control sobre el correcto asentamiento de la restauración, mayor estabilidad de color e incremento de las propiedades mecánicas ${ }^{(7,8)}$. La desventaja que presentan es que la luz para activarlas no llegue a todos los sitios, dificultando la polimerización de estos materiales.

Ehrmantraut y Bader midieron experimentalmente la profundidad de polimerización de resinas compuestas al ser iluminadas a través de distintos grosores de tejido dentario con tiempos de activación de 40 y 60 segundos. Ellos demostraron que la interposición de un material entre la fuente lumínica foto-activadora (luz halógena) y una resina compuesta afecta la profundidad de polimerización y, que el tiempo de exposición a la luz influye significativamente en la profundidad de polimerización, siendo ésta mayor al iluminar por más tiempo ${ }^{(5,8)}$.

1. CDDS. Departamento de Cirugía. Facultad de Odontología, Universidad de Chile. Chile.

2. Materiales Dentales. Departamento de Odontología Restauradora. Facultad de Odontología, Universidad de Chile. Chile. 
Es así que evaluar la profundidad de polimerización del elemento de fijación adquiere importancia, ya que si es escasa o nula quedará material sin polimerizar, que puede ser tóxico para las estructuras vivas debido a la mayor cantidad de monómero residual presente pudiendo potencialmente causar reacciones biológicas adversas y además las propiedades mecánicas del elemento de fijación serán deficientes, disminuyendo la vida útil de la restauración indirecta realizada con cerámica ${ }^{(5,10,11,12)}$.

Por estas razones señaladas anteriormente, es que nos planteamos la hipótesis que existen diferencias significativas en la profundidad de polimerización de resina compuesta fluida al interponer bloques de porcelana para estructuras libres de metal de distintos grosores.

El objetivo de este trabajo es determinar la profundidad de fototpolimerización de muestras de resina compuesta fluida al interponer bloques de porcelana para estructuras libres de metal de distintos grosores y distinta opacidad, según su contenido de relleno de óxidos metálicos, utilizando la emisión de luz de una lámpara de tipo LED, iluminando los distintos bloques de porcelana por un tiempo de 60 seg. con la resina fluida colocada en un formador de probetas de medida establecida debajo del material cerámico y establecer diferencias significativas entre los grupos en estudio.

De esta manera, se simula la polimerización de la resina compuesta que se utiliza como medio de fijación para restauraciones indirectas realizadas con cerámica.
Tabla 1. Características de los bloques de cerámicas utilizados en este estudio.

\begin{tabular}{|c|c|c|c|c|c|c|}
\hline $\begin{array}{c}\text { Grupos en } \\
\text { Estudio }\end{array}$ & $\begin{array}{c}\text { Marca } \\
\text { Comercial }\end{array}$ & Grosor & Manufactura & Tipo & Relleno & $\begin{array}{c}\text { Resistencia } \\
\text { Flexural }\end{array}$ \\
\hline 1 & Mark II & $3 \mathrm{~mm}$ & Vita, Alemania & Feldespática & $\begin{array}{l}\text { Partículas } \\
\text { vítreas }\end{array}$ & $120 \mathrm{mpa}$ \\
\hline 2 & Mark II & $4 \mathrm{~mm}$ & Vita, Alemania & Feldespática & $\begin{array}{l}\text { Partículas } \\
\text { vítreas }\end{array}$ & $120 \mathrm{mpa}$ \\
\hline 3 & Finesse & $3 \mathrm{~mm}$ & Denstply, USA & $\begin{array}{c}\text { Feldespática } \\
\text { con relleno }\end{array}$ & $\begin{array}{l}\text { Leucita } \\
(40-55 \%)\end{array}$ & $125 \mathrm{mpa}$ \\
\hline 4 & Finesse & $4 \mathrm{~mm}$ & Dentsply, USA & $\begin{array}{c}\text { Feldespática } \\
\text { con relleno }\end{array}$ & $\begin{array}{l}\text { Leucita } \\
(40-55 \%)\end{array}$ & $125 \mathrm{mpa}$ \\
\hline 5 & IPS emax & $3 \mathrm{~mm}$ & $\begin{array}{c}\text { Ivoclar, } \\
\text { Lichtentein }\end{array}$ & $\begin{array}{c}\text { Vitro- } \\
\text { cerámica }\end{array}$ & $\begin{array}{c}\text { Disilicato } \\
\text { de litio } \\
70 \%\end{array}$ & 400mpa \\
\hline 6 & IPS emax & $4 \mathrm{~mm}$ & $\begin{array}{c}\text { Ivoclar, } \\
\text { Lichtentein }\end{array}$ & $\begin{array}{c}\text { Vitro- } \\
\text { cerámica }\end{array}$ & $\begin{array}{c}\text { Disiliicato } \\
\text { de litio } \\
70 \%\end{array}$ & $400 \mathrm{mpa}$ \\
\hline 7 Cercon & 7 Cercon & $1.5 \mathrm{~mm}$ & Dentsply, USA & Policristalina & $\begin{array}{c}\text { Óxido de } \\
\text { zirconio } \\
92 \%\end{array}$ & $1200 \mathrm{mpa}$ \\
\hline 8 Cercon & 8 Cercon & $3 \mathrm{~mm}$ & Dentsply, USA & Policristalina & $\begin{array}{l}\text { Óxido de } \\
\text { zirconio } \\
92 \%\end{array}$ & $1200 \mathrm{mpa}$ \\
\hline 9 & 8 Control & $3 \mathrm{~mm}$ & Dentsply, USA & Policristalina & $\begin{array}{c}\text { Óxido de } \\
\text { zirconio } \\
92 \%\end{array}$ & $1200 \mathrm{mpa}$ \\
\hline
\end{tabular}

\section{MATERIALES Y MÉTODOS}

Se confeccionaron 8 especímenes de cerámicas para estructuras libres de metal de 3 y $4 \mathrm{~mm}$ de altura por $7 \mathrm{~mm}$ de diámetro de las marcas: Mark II (Vita), Finesse All-Ceramic (Dentsply) e IPS e.max Press (Ivoclar Vivadent) y de 1.5 y $3 \mathrm{~mm}$ de altura por $7 \mathrm{~mm}$ de diámetro de la marca Cercon (Dentsply), este menor espesor se relaciona con su alta opacidad ${ }^{(1,2,13)}$, obteniendo 8 grupos de estudio.

A través de estos bloques de porcelana se iluminó resina compuesta fluida Wave (SDI), que fue depositada en un formador metálico con perforaciones de $4 \mathrm{~mm}$ de alto por $6 \mathrm{~mm}$ de diámetro.

El material a base de resina se depositó al interior del formador metálico hasta el llenado total de éste, se cubrió la resina con un cubre objetos, sobre él se ubicó el bloque de porcelana correspondiente y se aplicó luz por sesenta segundos con lámpara LED Radi plus (SDI, Australia) la cual estuvo en contacto con el bloque cerámico durante toda la iluminación (Figura 1). La lámpara se calibró con Radiómetro LED (SDI, Australia) antes de iniciar el experimento y al finalizarlo. Como indicador adicional del funcionamiento de la lámpara y cada cinco cilindros polimerizados se controló que estuviera en un rango aceptado, que se comprueba al colocar la lámpara sobre el dispositivo el cual debe encender las cuatro luces que presenta.

Se retiraron los cilindros de resina compuesta obtenidos, se eliminó el material no polimerizado con espátula plástica, como lo indica la norma ISO 4049 para materiales de obturación y cementación a base de resina ${ }^{(14)}$ y se efectuó la medición individual de la altura de los cilindros, con un calibrador digital Lizt@ (Alemania) con sensibilidad de $0.01 \mathrm{~mm}$.

En total se confeccionaron 168 cilindros de resina compuesta, 20 por cada uno de los 8 bloques cerámicos descritos anteriormente y como grupo control positivo, se confeccionaros 8 cilindros de resina compuesta fluida los cuales fueron iluminados por $40 \mathrm{seg}$. interponiendo solamente un cubre objetos entre la resina y la fuente lumínica.

Obtenidos los datos para cada uno de los grupos, se tabularon y se registró su media, desviación estándar (DS) y error estándar de medida (SEM). Posteriormente, se utilizó el programa SPSS Statics versión (IBM) y se sometieron los valores obtenidos al test de ShapiroWilk y el test de homogeneidad de Levene para determinar distribución y homogeneidad de los mismos. Posteriormente, se aplicó el test de ANOVA (one-way) para determinar diferencia entre los grupos y se aplicó el test de Bonferroni para determinar los grupos que presentan diferencias (Tabla 1).

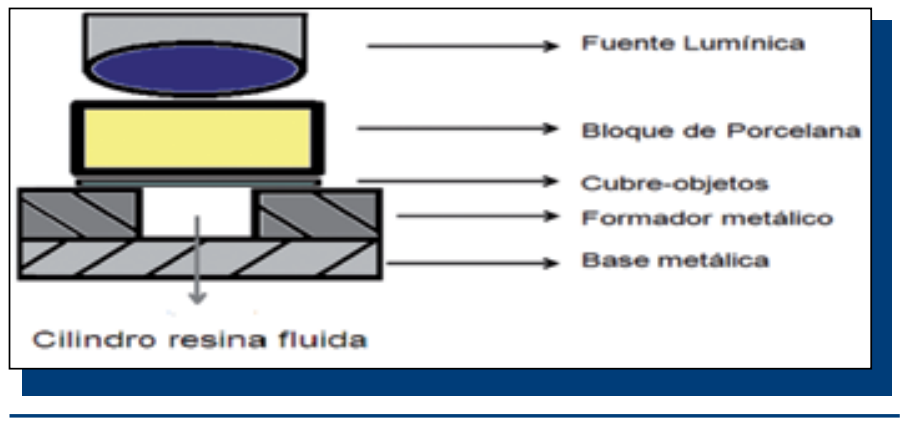

Figura 1. Esquema de polimerización de resina a través de bloques cerámicos.

\section{RESULTADOS}

Los datos se distribuyen en forma normal de acuerdo al test de Shapiro-Wilk tal como se muestran en la Tabla 2 (Figura 2).

Al realizar el test de homogeneidad de Levene, se demuestra que los resultados son homogéneos al obtener un valor superior a 0.05 como se muestra en la Tabla 3.

Se puede apreciar que existe diferencia significativa entre los grupos al aplicar el test one-way ANOVA como se muestra en la Tabla 4

Se procedió a la corrección estadística para múltiples observaciones de Bonferroni, comparando los grupos entre sí para determinar que grupos presenta diferencias entre ellos como se muestra en la Tabla 5.

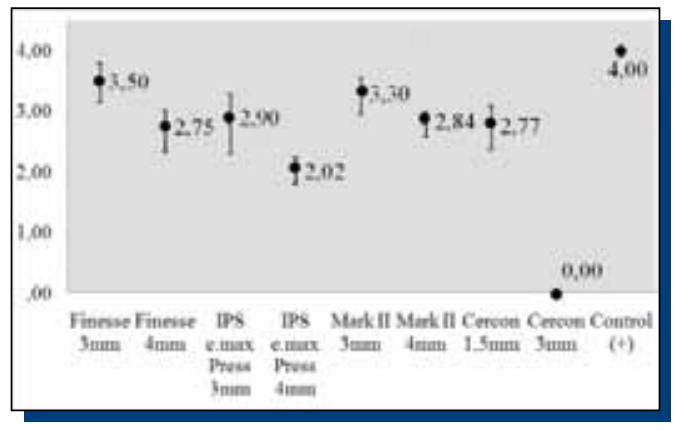

Figura 2. Valores de profundidad de polimerización, separados por grupo, expresados en $\mathrm{mm}$ 
Tabla 2. Valores de la Profundidad de Polimerización $(\mathrm{mm})$ para cada grupo y significancia con el test de Shapiro-Wilk.

\begin{tabular}{|l|c|c|c|c|c|c|c|}
\hline Porcelanas & $\mathbf{N}$ & Media & DS & SEM & Mín & Máx & $\begin{array}{c}\text { Significancia } \\
\text { Shapiro-Wilk }\end{array}$ \\
\hline Finesse 3mm & 20 & 3.4970 & .14371 & .03214 & 3.14 & 3.80 & .499 \\
\hline Finesse 4mm & 20 & 2.7505 & .19890 & .04448 & 2.32 & 3.01 & .168 \\
\hline $\begin{array}{l}\text { IPS e max } \\
\text { Press 3mm }\end{array}$ & 20 & 2.9020 & .22400 & .05009 & 2.29 & 3.29 & .258 \\
\hline $\begin{array}{l}\text { IPS e max } \\
\text { Press 4mm }\end{array}$ & 20 & 2.0175 & .12358 & .02763 & 1.80 & 2.23 & .270 \\
\hline Mark II 3mm & 20 & 3.2985 & .16014 & .03581 & 2.95 & 3.55 & .167 \\
\hline Mark II 4mm & 20 & 2.8435 & .09472 & .02118 & 2.58 & 2.99 & .123 \\
\hline $\begin{array}{l}\text { Cercon } \\
\text { 1.5mm }\end{array}$ & 20 & 2.7685 & .18207 & .04071 & 2.37 & 3.09 & .769 \\
\hline Cercon 3mm & 20 & .0000 & .00000 & .00000 & .00 & .00 & .314 \\
\hline Control (+) & 8 & 4.0038 & .02200 & .00778 & 3.97 & 4.03 & .03 \\
\hline Total & 168 & $\mathbf{2 . 5 8 0 8}$ & $\mathbf{1 . 0 7 0 1 4}$ & .08256 & .00 & $\mathbf{4 . 0 3}$ \\
\hline
\end{tabular}

Tabla 3. Test de Homogeneidad de Varianzas (Levene).

\section{Profundidad de Polimerización}

\begin{tabular}{|c|c|c|c|}
\hline Estadístico de Levene & df1 & df2 & Sig. \\
\hline 2.074 & 6 & 133 & .060 \\
\hline
\end{tabular}

Tabla 4. Test ANOVA.

\section{Profundidad de Polimerización}

\begin{tabular}{|c|c|c|c|c|c|}
\hline & $\begin{array}{c}\text { Sumas } \\
\text { Cuadráticas }\end{array}$ & df & $\begin{array}{c}\text { Medias } \\
\text { Cuadráticas }\end{array}$ & $\mathbf{F}$ & Sig. \\
\hline Entre Grupos & 26.596 & 6 & 4.433 & 160.411 & .000 \\
\hline $\begin{array}{c}\text { Dentro de los } \\
\text { Grupos }\end{array}$ & 3.675 & 133 & .028 & & \\
\hline Total & $\mathbf{3 0 . 2 7 1}$ & $\mathbf{1 3 9}$ & & & \\
\hline
\end{tabular}

Tabla 5. Comparación con test de Bonferroni.

\begin{tabular}{|c|c|c|c|c|c|c|c|c|}
\hline Grupos & $\begin{array}{c}\text { Mark II } \\
3 \mathrm{~mm}\end{array}$ & $\begin{array}{c}\text { Mark II } \\
4 \mathrm{~mm}\end{array}$ & $\begin{array}{c}\text { Finesse } \\
3 \mathrm{~mm}\end{array}$ & $\begin{array}{c}\text { Finesse } \\
4 \mathrm{~mm}\end{array}$ & $\begin{array}{l}\text { IPS max } \\
3 \mathrm{~mm}\end{array}$ & $\begin{array}{l}\text { IPS max } \\
4 \mathrm{~mm}\end{array}$ & $\begin{array}{l}\text { Cercon } \\
1.5 \mathrm{~mm}\end{array}$ & $\begin{array}{c}\text { Cercon } \\
3 \mathrm{~mm}\end{array}$ \\
\hline $\begin{array}{l}\text { Mark II } \\
3 \mathrm{~mm}\end{array}$ & ------- & $0.000^{*}$ & $0.002^{*}$ & $0.000^{*}$ & $0.000^{*}$ & $0.000^{*}$ & $0.000^{*}$ & $0.000^{*}$ \\
\hline $\begin{array}{c}\text { Mark II } \\
4 \mathrm{~mm}\end{array}$ & $0.002^{*}$ & ------ & $0.000^{*}$ & 1.000 & 1.000 & $0.000^{*}$ & 1.000 & $0.000^{*}$ \\
\hline $\begin{array}{c}\text { Finesse } \\
3 \mathrm{~mm}\end{array}$ & $0.002^{*}$ & $0.000^{*}$ & -------- & $0.000^{*}$ & $0.000^{*}$ & $0.000^{*}$ & $0.000^{*}$ & $0.000^{*}$ \\
\hline $\begin{array}{c}\text { Finesse } \\
4 \mathrm{~mm}\end{array}$ & $0.000^{*}$ & 1.000 & $0.000^{*}$ & ------- & 0.070 & $0.000^{*}$ & $1.000^{*}$ & $0.000^{*}$ \\
\hline $\begin{array}{l}\text { IPS max } \\
3 \mathrm{~mm}\end{array}$ & $0.000^{*}$ & 1.000 & $0.000^{*}$ & 0.070 & ------- & $0.000^{*}$ & 0.222 & $0.000^{*}$ \\
\hline $\begin{array}{l}\text { IPS max } \\
4 \mathrm{~mm}\end{array}$ & $0.000^{*}$ & $0.000^{*}$ & $0.000^{*}$ & $0.000^{*}$ & $0.000^{*}$ & ------ & $0.000^{*}$ & $0.000^{*}$ \\
\hline $\begin{array}{l}\text { Cercon } \\
1.5 \mathrm{~mm}\end{array}$ & $0.002^{*}$ & 1.000 & $0.000^{*}$ & 1.000 & 0.222 & $0.000^{*}$ & ------ & $0.000^{*}$ \\
\hline $\begin{array}{c}\text { Cercon } \\
3 \mathrm{~mm}\end{array}$ & $0.002^{*}$ & $0.000^{*}$ & $0.000^{*}$ & $0.000^{*}$ & $0.000^{*}$ & $0.000^{*}$ & $0.000^{*}$ & ------ \\
\hline Control & $0.000^{*}$ & $0.000^{*}$ & $0.000^{*}$ & $0.000^{*}$ & $0.000^{*}$ & $0.000^{*}$ & $0.000^{*}$ & $0.000^{*}$ \\
\hline
\end{tabular}

\section{DISCUSIÓN}

La resina compuesta Wave fue polimerizada en una profundidad menor que el alto del cilindro en la que se depositó el material, es decir que en ningún grupo en estudio se obtuvo polimerización de $4 \mathrm{~mm}$, al ser iluminadas a través de bloques cerámicos, con una lámpara led, por 60 seg y en el grupo en que se utilizó cerámica Cercon en grosor de $3 \mathrm{~mm}$ la polimerización fue nula. En este tiempo de iluminación, todas las porcelanas permitieron el paso de luz, incluyendo a la Cercon, en grosor de $1.5 \mathrm{~mm}$, que presenta opacidad muy alta, cercana a un metal(1,2,13), que logró endurecer la resina compuesta que se encontraba subyacente a una profundidad de $2.77 \mathrm{~mm}$, aunque este valor es superior al grupo de porcelana IPS emax de $4 \mathrm{~mm}$ de espesor, que polimerizó $2.02 \mathrm{~mm}$, pero es un material de menor opacidad. Este tiempo es superior al que se utiliza para activar las resinas de restauración directa en grosores de $3 \mathrm{~mm}$ y es recomendado por los fabricantes de dichos materiales ${ }^{(15)}$. El mismo tiempo se utiliza para activar aquellos materiales a base de resina compuesta de activación dual, que se utilizan para fijar restauraciones indirectas que superen los $6 \mathrm{~mm}$ de espesor.

Los grupos Mark II 4mm, Finesse $4 \mathrm{~mm}$, IPS e.max Press $3 \mathrm{~mm}$ y Cercon $1.5 \mathrm{~mm}$, no presentaron diferencias estadísticamente significativas entre ellos y estas cerámicas están aquí ordenadas ascendentemente en su resistencia flexural y su opacidad. Los grosores utilizados en este estudio son similares a los que se obtienen en restauraciones indirectas en aquellas zonas de cajones proximales o en zonas de mayor destrucción de la pieza dentaria a tratar por lo que el paso de la luz no se interrumpe, permitiendo la activación y posterior polimerización de la resina subyacente, que se utiliza como agente de fijación.

Es de relevancia analizar los grupos que no presentaron diferencias significativas entre sí (grupo 2, 2.84mm+/-0.09; grupo 4, 2.75mm+/-0.19; grupo $5,2.9 \mathrm{~mm}+/-0.22$ y grupo $7,2.77 \mathrm{~mm}+/-0.18$ ), ya que se establece un punto de equivalencia de profundidad de polimerización para cerámicas distintas en resistencia flexural y en estructura; la translucidez de estas porcelanas no es igual por el distinto relleno y el porcentaje de éste que presentan. Por las distintas características de translucidez de estas cerámicas, sus grosores deben ser diferentes para que pueda polimerizar el mismo grosor de resina fluida subyacente.

Heffernan MJ y cols., evaluaron la cantidad de luz que es capaz de traspasar la cerámica, pero no con el propósito de establecer la profundidad de polimerización que pueda lograr un agente de fijación (cemento a base de resina compuesta). Los mismos autores señalan que el óxido de circonio tiene opacidad similar a un metal, que la luz no traspasa a este material ${ }^{(1,2)}$. Este resultado se contrapone con este estudio, ya que demuestra que la luz de una lámpara LED es capaz de activar la polimerización de resinas compuesta fluida, al ser iluminada a través de cerámicas en base a óxidos de circonio en grosores de $1.5 \mathrm{~mm}$.

En oposición a Heffernan, Baldissara, al estudiar la trans-iluminación de elementos a base de cerámicas, encuentra diferencias significativas, aunque observó que en todas las cerámicas la luz las traspasó(16).

Al aumentar el grosor de la porcelana se genera reducción exponencial de la intensidad de la luz, medidos en grosores de hasta $4 \mathrm{~mm}$. En un estudio de Lee IB et al., la intensidad de la luz llegó a valores cercanos a $0 \mathrm{~mW} / \mathrm{cm}^{2}$ cuando el grosor de la porcelana fue de $4 \mathrm{~mm}$, sin embargo, la fuente de luz utilizada fue una de tipo halógena que generaba valores de 500 $\mathrm{mW} / \mathrm{cm}^{2(17)}$, de inferior potencia que la LED $\left(\mathrm{mW} / \mathrm{cm}^{2}\right)$. En el presente estudio fue posible polimerizar resina a través de grosores de $4 \mathrm{~mm}$ de cerámica, esto puede 
ser atribuido a la tecnología diferente utilizada en la fuente emisora de luz, una lámpara LED de segunda generación que presenta mayor valor expresado en $\mathrm{mW} / \mathrm{cm}^{2}$.

La literatura reporta que en grosores de restauraciones mayores de $5 \mathrm{~mm}$ no es posible lograr foto activación, por lo que es necesario materiales de activación dual, pero en este tipo de elementos de fijación, los agentes foto-iniciadores pueden interactuar con los agentes auto-polimerizadores, inhibiendo la completa polimerización ${ }^{(18)}$. Esto puede ser compensado esperando 5-10 minutos antes de aplicar luz, ya que así se permitirá el correcto accionar del auto-polimerizador ${ }^{(19)}$. Este tiempo utilizado en clínica resulta demasiado largo para una fijación.

El uso de los cementos de activación dual se indica en aquellas situaciones en que la luz no es capaz de penetrar el material de restauración para activar el cemento y producir su polimerización, pero con las lámparas tipo LED, se ha comprabado que son capaces de atravezar estas estructuras y polimerizar el material de cementación con tiempos que no superan los 60 seg., y que representa un tiempo menor al que se necesita para que aquellos de curado dual aseguren un polimerizado adecuado(11).

El uso de los cementos de foto-curado está limitado a situaciones como la cementación de carillas o incrustaciones de poco grosor, en donde éste o el color de la restauración no afecten la habilidad de la luz activadora para polimerizar el cemento ${ }^{(19,20)}$.

Es importante reevaluar los cementos de resina de fotopolimerización como medio de cementación y bajo qué circunstancias pueden ser utilizados, ya que con las actuales fuentes de iluminación es posible obtener mayor energía que es capaz de atravesar grosores de cerámica de hasta $4 \mathrm{~mm}$ que se utilizan en restauraciones libres de metal.

\section{CONCLUSIONES}

Existen diferencias significativas en la profundidad de polimerización de resina compuesta fluida al interponer bloques de cerámica para estructuras libres de metal de distintos grosores, siendo los mayores grosores los que dificultan en mayor medida el paso de la luz.

La composición y estructura de las cerámicas para prótesis dentales libres de metal interfieren en la profundidad de fotopolimerización de resina fluida, siendo más eficaces en dificultar el paso de la luz aquellas que presentan mayor contenido de relleno de óxidos metálicos y más aún la que son de estructura poli cristalina, como lo es la cerámica Cercon.

De acuerdo a este estudio es posible foto-polimerizar resina fluida a través de bloques de cerámica para estructuras libres de metal, a excepción de la cerámica policristalina de zirconio Cercon (Dentsply) de $3 \mathrm{~mm}$ de grosor, por lo que el grosor del zirconio influye significativamente en la profundidad de foto-polimerización respecto a las otras cerámicas estudiadas.

El uso de resinas compuestas fluidas como medio de fijación de restauraciones cerámicas libres de metal es posible, ya que la profundidad de polimerización que se obtiene en este estudio es mayor que el grosor que se establece la norma ISO $\mathrm{N}^{\circ}$ 4049:2000 para la interface diente restauración para este tipo de materiales. Según esta Norma ISO, la línea de cementación de un cemento en base a resina compuesta no debe ser mayor a 50 micrones $^{(18)}$.

\section{CONFLICTO DE INTERESES} ningún tipo.
Los autores declaran no presentar conflictos de interés de

\section{REFERENCIAS BIBLIOGRÁFICAS}

1. Heffernan MJ et al. Relative translucency of six all-ceramic systems. Part II: Core and veneer materials. J Prosthet Dent, 2002; 88(1): 10-15.

2. Heffernan MJ et al. Relative translucency of six all-ceramic systems. Part I: Core materials. J Prosthet Dent, 2002; 88(1): 4-9.

3. Ferracane JL. Resin composite-State of the art. Dent Mater, 2011; 27(1): 29-38. 4. Ferracane JL. Current trends in dental composites. Crit Rev Oral Biol Med, 1995; 6(4): 302-318.

5. Ehrmantraut M, Bader M. Efecto de la interposición de vidrio ionómero en la polimerización de resinas compuestas a traves de estructuras dentarias. Rev Fac Odontol Univ Chile, 1996; 14(1): 49-55.

6. Kwon TY, Bagheri R, Kim YK, Kim KH, Burrow MF. Cure mechanisms in materials for use in esthetic dentistry. J Investig Clin Dent, 2012; 3(1): 3-16.

7. Attar N, Tam LE, McComb D. Flow, strength, stiffness and radiopacity of flowable resin composites. J Can Dent Assoc, 2003; 69(8): 516-521.

8. Barceleiro $\mathrm{M}$ de $\mathrm{O}$ et al. Shear bond strength of porcelain laminate veneer bonded with flowable composite. Oper Dent, 2003; 28(4): 423-428.

9. Ehrmantraut M, Bader M. Polimeración de resinas compuestas a través de estructuras dentarias. Rev Fac Odontol Univ Chile, 1994; 12(2): 22-27.

10. Ozyesil AG, Usumez A, Gunduz B. The efficiency of different light sources to polymerize composite beneath a simulated ceramic restoration. J Prosthet Dent, 2004; 91(2): 151-157.

11. Caughman WF, Chan DC, Rueggeberg FA. Curing potential of dualpolymerizable resin cements in simulated clinical situations. J Prosthet Dent, 2001; 86(1): 101-106.
12. Krifka $S$ et al. Resin monomer-induced differential activation of MAP kinases and apoptosis in mouse macrophages and human pulp cells. Biomaterials, 2010; 31(11): 2964-2975.

13. Kelly JR. Dental ceramics: Current thinking and trends. Dent Clin North Am, 2004; 48(2): 513-530.

14. Moore BK et al. Depth of cure of dental resin composites: ISO 4049 depth and microhardness of types of materials and shades. Oper Dent, 2008; 33(4): 408-412.

15. http://www.sdi.com.au/images/stories/brouchers/brouchersES/lceBroES.pdf (visitado el 10/12/2012).

16. Baldissara $P$ et al. Translucency of zirconia copings made with different CAD/ CAM systems. J Prosthet Dent, 2010; 104(1): 6-12.

17. Lee IB et al. Influence of ceramic thickness and curing mode on the polymerization shrinkage kinetics of dual-cured resin cements. Dent Mater, 2008 24(8): 1141-1147.

18. Pereira SG et al. Effect of curing protocol on the polymerization of dual-cured resin cements. Dent Mater, 2010; 26(7): 710-718.

19. Manso AP et al. Cements and adhesives for all-ceramic restorations. Dent Clin North Am, 2011; 55(2): 311-332, IX.

20. Ferracane JL. Resin composite-State of the art. Dent Mater, 2011; 27(1): 29-38 